\section{Roundtable on neuroendocrinology}

\section{from Barend ter Haar}

During the weekend April 6-7 recent neuroendocrinological findings of most of the leading laboratories in Europe were discussed informally at Magdalen College, Oxford in an effort to establish the most fruitful course of future research, and to sow the seeds for greater international collaboration. The sessions were divided under the general headings of hypothalamic releasing hormones, secretion of the hormones of the posterior pituitary, monoamine systems affecting endocrine function, rhythms involved in neural control of reproductive function and processes involved in sexual differentiation and puberty.

It had been hoped that with the elucidation of the amino acid sequence of luteinising hormone releasing hormone (LHRH) and its subsequent chemical synthesis, antibodies prepared against this material could be used to identify, by immunofluorimetric techniques, the neurosecretory cells for LHRH in the brain. Though J. Barry (University of Lille) has been able to demonstrate a distribution of such neurones throughout certain areas of the anterior and medial hypothalamus of the guinea pig, he has as yet been relatively unsuccessful in other species: there is still much progress to be made in this field. It was noted with interest that Barry has also found labelled axons extending from the hypothalamus to the limbic system: it is now becoming more obvious that classical concepts of unidirectional flow of neuronal information from the limbic to the anterior hypothalamus and then to the median eminence have to be altered.

To add to the complexity, M. C. Harris (University of Birmingham) and G. B. Makara (Institute for Experimental Medicine, Budapest) indicated that they had also found not only cells in the arcuate nucleus and median eminence with axonal terminations in the anterior hypothalamus, but also hypothalamic cells which appeared to project both forwards, to the paraventricular nucleus, and also caudally, to the median eminence. Of further interest concerning efferents from the hypothalamus to other brain areas, and in the context of recent observations of effects of thyrotropin releasing hormone (TRH) on certain psychiatric disorders, were the observations presented by $\mathbf{R}$. Dyer (University of Bristol) that both TRH and LHRH can modify electrical activity in the cerebral cortex in a similar way to their observations in the hypothalamus.

It is clear that though radioimmuno- assay techniques have greatly helped to advance neuroendocrine knowledge, great care is still needed in the interpretation of results, the principal difficulty being that immunoreactive sites on a molecule do not necessarily relate to biologically active sites. Furthermore, such assay techniques are of little use in the detection of brief hormonal discharges such as the 2-second discharge of oxytocin demonstrated by D. W. Lincoln (University of Bristol) by means of transient changes in mammary pressure. For the release of posterior pituitary hormones, N. A. Thorn (University of Copenhagen) suggested a similar involvement of calcium in hormone discharge as in the mechanisms of muscle contraction.

In discussing the effect of circadian rhythms on reproductive function, $\mathbf{B}$. $\mathbf{K}$. Follett (University College of North Wales, Bangor) described how in the sparrow photosensitivity seems to vary in a circadian fashion in that a limited period of sensitivity occurs about $12 \mathrm{~h}$ after dawn. He has found that if light is present at this time reproductive activity is promoted so that sexual activity becomes linked with maximum day length. P. C. B. MacKinnon (University of Oxford) described how the circadian rhythm of cerebral protein metabolism in the rat is sexually differentiated, and put forward the hypothesis that the 'female' rhythm is involved in the synchronisation of internal and external signals to bring about reproductive activity in the most suitable conditions.

In considering the processes involved in the onset of puberty, F. Döcke (Humboldt University, Berlin) indicated that the limbic system is involved in controlling sexual development, and $\mathbf{H}$. Moll (Erasmus University, Rotterdam) suggested that the search for only one control process may be misguided, and that puberty may be brought about by the coordinated maturation of several processes.

Obviously much more was discussed than there is space here to relate. The organisational efforts of B. T. Donovan (Institute of Psychiatry, London), C. Kordon (INSERM, Paris), and G. P. van Rees (University of Leiden) in getting together so many authorities on various branches of neuroendocrinology were well rewarded by the informal exchange of ideas that was achieved.

\section{Correction}

IN the News and Views article "Beyond systems ecology" (Nature, 248,637 ; 1974), the authorship of the two publications cited was misattributed. The article in Proc. N.Y. Acad. Sci. is by $\mathbf{R}$. Levins, and the book Stability and Complexity in Model Ecosystems is by R. M. May.

\section{Characterising the stringent system}

from Benjamin Lewin

Molecular Genetics Correspondent

Possible relationships between protein synthesis and transcription have attracted attention for more than a decade, and in recent months this concentration of interest has been justified by results which may lead to the definition of the components of the stringent system of Escherichia coli. Genetic characterisation of this system has so far led to the isolation of two c'asses of mutant. As was first shown by Cashel and Gallant (Nature, 221, 838; 1969) and Cashel (J. biol. Chem., 244, 3133 ; 1969) relaxed $\left(\mathrm{rel}^{-}\right)$mutants do not restrain ribosomal RNA synthesis during starvation for required amino acids because (unlike the wild type stringent cells) they cannot increase their levels of the 'magic spot' compounds ppGpp and pppGpp in response to the deprivation. Cells of the spotless (spo $T^{-}$) mutant, isolated more recently by Laffler and Gallant (Cell, 1, 27; 1974), cannot produce pppGpp during amino acid starvation, but overproduce ppGpp.

Although it is clear that accumulation of ppGpp and pppGpp is responsible for inhibiting RNA synthesis, the mechanism of formation and action of these two compounds has only more recently come under investigation. By following the abilities of $\mathrm{rel}^{+}$and $\mathrm{rel}^{-}$ strains to form magic spots and respond to changes in growth conditions, Lazzarini, Cashel and Gallant (J. biol. Chem., 246, 4381; 1971) found that relcells, although unable to respond to amino acid starvation, show the same response as $\mathrm{rel}^{+}$cells to other changes, such as shifts in growth rate. The rel gene must therefore code for a protein implicated in forming $p p G p p$ and pppGpp specifically in response to lack of amino acids, a reaction which has proved to be mediated by the consequent lack of aminoacyl-tRNA. By showing that the stability of ppGpp is much enhanced in spot $T^{-}$cells, Stamminger and Lazzarini (Cell, 1, 85; 1974) have come to the conclusion that the spoT gene product may be responsible for converting $\mathrm{ppGpp}$ to $\mathrm{pppGpp}$. Clearly, many other genes may also be part of this system and the levels of the magic spots may be controlled at degradation as well as synthesis.

The concept originally suggested some time ago that an 'idling reaction' of protein synthesis may be responsible for formation of magic spots has recently been substantiated by the use of in vitro systems. Haseltine et al. (Nature, 238, 381; 1972) found that ribosomes from stringent cells can synthesise ppGpp and pppGpp in vitro 